\title{
Gender reassignment: 5 years of referrals in Oxfordshire
}

\author{
Kate Saunders, ${ }^{1}$ Christopher Bass ${ }^{2}$
}

The Psychiatrist (2011), 35, 325-327, doi: 10.1192/pb.bp.110.032664

${ }^{1}$ Warneford Hospital, Oxford; ${ }^{2}$ John Radcliffe Hospital, Oxford

Correspondence to Kate Saunders (kate.saunders@psych.ox.ac.uk)

First received 23 Sep 2010, final revision 23 Feb 2011, accepted 25 May 2011

\begin{abstract}
Aims and method To evaluate the characteristics of individuals seeking gender reassignment, the frequency of subsequent referrals to a specialist centre, and funding approval. Cases were identified from a local referrals database and data were extracted from case notes.

Results Fifty-four individuals attended for assessment; 70\% were biological males and $30 \%$ were biological females. Mean age at referral was significantly different between the two groups. Over half were taking hormone supplementation and three had already had surgery. Further, $24 \%$ had a current and $30 \%$ a past mental illness. The majority of individuals were referred to a specialist centre for gender reassignment but only two had funding for surgery approved. Paedophilia was a rare but concerning finding.
\end{abstract}

Clinical implications Psychiatric assessment plays an important role in confirming transsexualism and in identifying other relevant diagnoses.

Declaration of interest None.
Gender dysphoria is the personal experience of dissonance between actual and desired gender. When these feelings are persistent, the person is diagnosed with gender identity disorder. ${ }^{1}$ It is a rare disorder, associated with significant psychological distress. Treatment involving hormones and gender-confirming surgery has been associated with excellent social, sexual and psychological outcomes. ${ }^{2}$ In the UK the Gender Recognition Act 2004 allows individuals to obtain legal recognition of their post-transition gender. Lifetime psychiatric comorbidity is high in people with gender identity disorder, ${ }^{3}$ with $71 \%$ reported to have a current and/or lifetime Axis I disorder. ${ }^{1}$ The outcome of premorbid psychopathology following gender reassignment surgery is unclear. Mate-Kole et $a l^{2}$ reported improvements in sexual functioning and neurotic symptoms, whereas Udeze et $a l^{4}$ reported no significant effect on psychological functioning 6 months post-surgery. Following a successful appeal against a local health authority in $1999,{ }^{5}$ a blanket ban on funding of medical treatment for gender reassignment was deemed illegal and those diagnosed with gender identity disorder are entitled to appropriate treatment in the National Health Service (NHS). The courts have deemed genital surgery to be an appropriate treatment in this context. However, there are ongoing difficulties in accessing NHS-funded surgical procedures and geographical variation is wide. ${ }^{6}$

In Oxfordshire, treatment funding for core gender reassignment surgery is provided on an individual basis. The person must fulfil the current World Professional Association for Transgender Health criteria $^{7}$ and be recommended as suitable for surgery by a specialist NHS gender identity clinic. Cosmetic surgery and other procedures, such as breast surgery, larynx reshaping, rhinoplasty, hair removal, jaw reduction and waist liposuction, are considered 'low priority' and not a core part of gender reassignment surgery. These are not normally funded unless in exceptional circumstances, although the local primary care trust does not define 'exceptional' in this context.

All individuals who request treatment for gender dysphoria in Oxfordshire are referred to a single clinician (C.B.) for psychiatric assessment and subsequent referral to a specialist centre. There is currently no provision locally for ongoing specialist follow-up for those awaiting surgery. Referrals are received directly from primary care and from local psychiatric teams. We wanted to review the characteristics of individuals referred for assessment as well as the frequency of subsequent requests for surgery and funding approval.

\section{Method}

Individuals referred to C.B. between 2004 and 2009 were identified from a referral database kept by the Department of Psychological Medicine at the John Radcliffe Hospital in Oxford. Case notes were reviewed by C.B. and K.S. Data were extracted from the initial assessment notes and recorded on a form. Comparative analyses were then conducted using the student $t$-test for dimensional variables and the $\chi^{2}$-analysis for categorical variables was performed using the statistical package SPSS version 17.0 on Windows 7. 


Table 1 Axis I and II morbidity
\begin{tabular}{lccc} 
& & & \\
& Male to female $(N=38)$ & Female to male $(N=16)$ & Total $(N=54)$ \\
\hline Age, years: mean (s.d.) & $35.1(14.2)$ & $26.9(9.6)^{\star}$ & $32.2(13.4)$ \\
\hline Axis I disorder, $n$ (\%) & & & $13(24)$ \\
Current & $8(28)$ & $7(43)$ & $16(30)$ \\
Past & $9(24)$ & & $16(3)$ \\
\hline
\end{tabular}

${ }^{\star} P=0.018$.

\section{Results}

Fifty-four individuals from a total of 56 referrals received attended for assessment. There were significantly more individuals seeking male-to-female transition $(70 \%, n=39)$ than those seeking female-to-male transition $(30 \%, n=15)$. The biological females were significantly younger than their male counterparts at referral (Table 1).

The majority $(51 \%, n=29)$ were referred for initial assessment as opposed to follow-up or re-evaluation of their eligibility for specialist referral. Eleven males (29\%) and six females $(40 \%)$ were taking hormone supplementation: eight males (21\%) were taking oestrogens, one was taking oestrogens and antiandrogen, one was taking a gonadotropin-releasing hormone $(\mathrm{GnRH})$ analogue, and one a 5-alpha reductase inhibitor; of females, five (27\%) were taking testosterone (four depot and 1 gel) and one was taking a GnRH agonist. Two males (3\%) had a penectomy, orchidectomy and vaginoplasty; two females (13\%) had already had a mastectomy and one a phalloplasty.

Over half of individuals taking hormone supplementation had purchased it on the internet and were not receiving any form of physical monitoring for adverse effects. Of the whole sample, $24 \%(n=13)$ had a current Axis I disorder and $30 \%(n=16)$ had a history of mental illness. No significant differences in past or current mental illness were found between the biological males and biological females. Depression was the most common current and past diagnosis (Fig. 1).

The majority of individuals $(80 \%, n=45)$ were referred to a specialist centre for gender reassignment (Fig. 2), although only two (4\%) have had funding for surgery approved at the time of the study. Three of the sample had or were planning to have surgery abroad, on the basis that

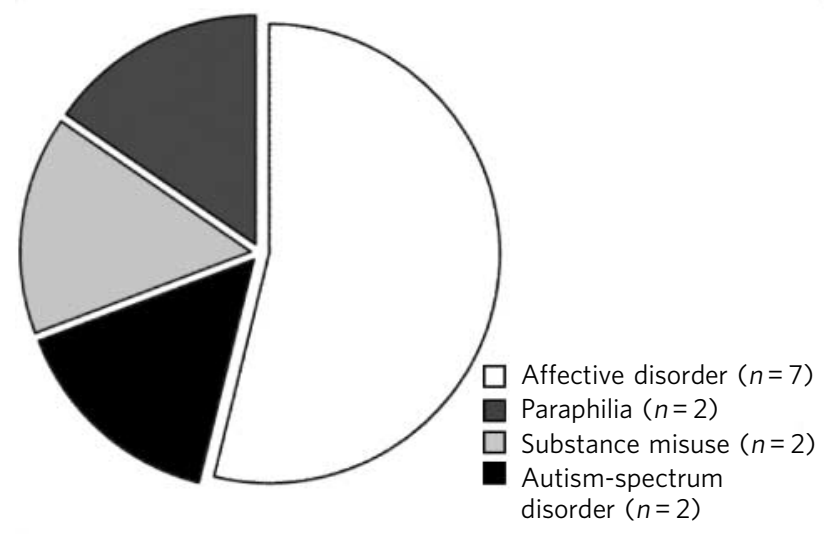

Fig 1 Current Axis I disorders in the sample. services there were more easily accessible and less expensive than in the UK. Reasons for non-referral to a specialist centre included being deemed not ready for transitioning (either determined by the individual or because the person was not currently living in the desired gender role), being homosexual but not having gender identity disorder, having an autism-spectrum disorder with a significant degree of impairment such that the real-life experience criterion was not met, and seeking gender reassignment to facilitate or normalise paedophilia. This latter small group described gender reassignment as a means by which to increase their intimate contact with children, which they viewed to be more socially acceptable in a female role.

\section{Discussion}

The results of our study are broadly consistent with the current literature. The younger age of those seeking femaleto-male transition has been described in a number of studies. ${ }^{8,9}$ Levels of psychiatric comorbidity appear to be lower than those described by Hepp and colleagues. ${ }^{3}$ The relationship between autism-spectrum disorders and gender dysphoria has been described by a number of authors. ${ }^{10-12}$ It poses a significant challenge given the social and communication difficulties associated with this diagnosis. This becomes a particular issue as living and functioning in

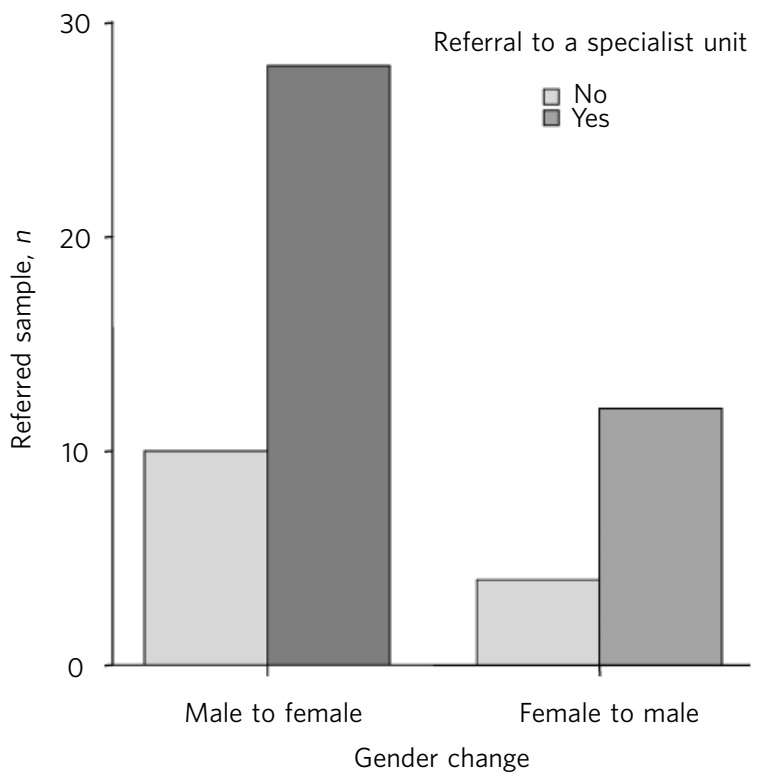

Fig 2 Referral to a specialist centre. 
role are usually prerequisites for referral for surgery. There are also issues of capacity to consent to treatment, particularly if the presence of an autism-spectrum disorder is associated with intellectual disabilities, where it is advised that psychological and social treatments be pursued. ${ }^{11}$ We have been unable to identify any previous literature describing individuals seeking gender reassignment as a means of normalising their paedophilia, but this is clearly a concerning finding.

Another worrying finding is that the internet has provided a new means by which to acquire drug treatments without seeing a doctor. Although this allows greater privacy and ease of access to hormone treatment, the chemical composition of medications acquired on the internet is often uncertain and any contraindications, interactions or side-effects may go unchecked.

The barriers faced by individuals seeking gender reassignment are considerable. There is widespread variability in the services funded by local primary care trusts, ${ }^{7}$ and the proposed changes to NHS commissioning in England are likely to complicate this issue further. The lengthy process is often cited as a contributory factor in the development of comorbid psychiatric problems, and untreated gender dysphoria can be associated with significant psychological distress and is a risk factor for suicide. $^{2}$ The costs of treatment need to be weighed up against the ongoing costs of supporting the mental healthcare needs of those awaiting genital surgery.

\section{Limitations}

This study is retrospective in nature and its primary objective was not to establish psychiatric diagnoses. None of the individuals seen by the service had a formal diagnostic interview so it is likely that Axis II comorbidity in this sample is underreported. Oxfordshire has a high student population, with two universities in the city, which may have introduced some bias.

\section{Future direction of services}

Psychiatric comorbidity in the Oxfordshire cohort was lower than that described in other samples, but despite many individuals meeting the appropriate criteria, funding for surgery was rare. In view of this there is a case for ongoing psychological support to be provided for individuals awaiting surgery. Doctors should routinely enquire about where hormones are being bought from, advise against online purchasing and ensure appropriate physical health monitoring.

\section{About the authors}

Kate Saunders, MRCPsych, is OHSRC Junior Fellow, University Department of Psychiatry, Warneford Hospital, Oxford, and Christopher Bass, MD FRCPsych, is Consultant Liaison Psychiatrist, John Radcliffe Hospital, Oxford.

\section{References}

1 American Psychiatric Association. Diagnostic and Statistical Manual of Mental Disorders (4th edn, text revision) (DSM-IV-TR). APA, 2000.

2 Mate-Kole C, Freschi M, Robin A. A controlled study of psychological and social change after surgical gender reassignment in selected male transsexuals. Br J Psychiatry 1990; 157: 261-4.

3 Hepp U, Kraemer B, Schnyder U, Miller N, Delsignore A. Psychiatric comorbidity in gender identity disorder. J Psychosom Res 2005; 58: 259-61.

4 Udeze B, Abdelmawla D, Khoosal D, Terry T. Psychological functions in male-to-female transsexual people before and after surgery. Sexual Relationship Therapy 2008; 23: 141-5.

5 Appeals Court judgement of North-West Lancashire Health Authority $v$ A, D and G (QBC 1999/0226/4; 0228/4; 0230/4)

6 Murjan S, Shepherd M, Ferguson BG. What services are available for the treatment of transsexuals in Great Britain? Psychiatr Bull 2002; 26: 210-2.

7 Meyer W, Bockting WO, Cohen-Kettenis P, Coleman E, DiCeglie D, Devor $\mathrm{H}$, et al. The Harry Benjamin International Gender Dysphoria Association's Standards of Care for Gender Identity Disorders, Sixth Version. World Professional Association for Transgender Health, 2001.

8 Dixen JM, Maddever H, Van Maasdam J, Edwards PW. Psychosocial characteristics of applicants evaluated for surgical gender reassignment. Arch Sex Behav 1984; 13: 269-76.

9 Blanchard R, Clemmensen LH, Steiner BW. Heterosexual and homosexual gender dysphoria. Arch Sex Behav 1987; 16: 139-52.

10 de Vries ALC, Noens ILJ, Cohen-Kettenis PT, van Berckelaer-Onnes IA Doreleijers TA. Autism spectrum disorders in gender dysphoric children and adolescents. J Autism Development Disord 2010; 40: 930-3.

11 Parkes G, Hall I. Gender dysphoria and cross-dressing in people with intellectual disability: a literature review. Ment Retard 2006; 44: 260-71.

12 Landén M, Rasmussen P. Gender identity disorder in a girl with autism a case report. Eur Child Adolesc Psychiatry 1997; 6: 170-3. 